\title{
Additional articles of special issue on Real-time image watermarking and forensics in cloud computing
}

\author{
Chuan Qin ${ }^{1} \cdot$ Zhenxing Qian ${ }^{2} \cdot$ Guorui Feng ${ }^{3} \cdot$ Xinpeng Zhang ${ }^{2}$
}

Published online: 1 July 2019

○) Springer-Verlag GmbH Germany, part of Springer Nature 2019

As an addition to the special issue on Real-Time Image Watermarking and Forensics in Cloud Computing which was published in volume 16, issue 3, June 2019 (https:// link.springer.com/journal/11554/16/3), an overview of five additional papers is provided in this guest editorial. These papers address the following areas: (1) image watermarking (one paper), (2) reversible data hiding (three papers), and (3) emerging technique (one paper).

\section{Image watermarking}

The paper entitled "FPGA-Based Low-Complexity HighThroughput Real-Time Hardware Accelerator for Robust Watermarking", co-authored by Hangqi Ge and Jin Sha, proposes an FPGA-based hardware accelerator for robust image watermarking, which simplifies the DCT/IDCT algorithms and only involves the addition and shift operations instead of the complex multiplication/division and nonlinear function. The proposed algorithm significantly reduces the hardware complexity and enhances the throughput by a pipelined implementation. Experimental results show that

Chuan Qin

qin@usst.edu.cn

Zhenxing Qian

zxqian@fudan.edu.cn

Guorui Feng

grfeng@shu.edu.cn

Xinpeng Zhang

zhangxinpeng@fudan.edu.cn

1 School of Optical-Electrical and Computer Engineering, University of Shanghai for Science and Technology, Shanghai 200093, China

2 School of Computer Science, Fudan University, Shanghai 200433, China

3 School of Communication and Information Engineering, Shanghai University, Shanghai 200444, China the proposed accelerator has good efficiency and excellent performance achieving robust image watermarking.

\section{Reversible data hiding}

The paper entitled "Adaptive Error Prediction Method Based on Multiple Linear Regression for Reversible Data Hiding", co-authored by Bin Ma, Xiaoyu Wang, Qi Li, Bing Li, Jian $\mathrm{Li}$, Chunpeng Wang, and Yunqing Shi, presents a new type of error prediction method based on multiple linear regression (MLR) algorithm, in which the objected pixel can be estimated though MLR function and its surrounding pixels according to the consistency of the pixels in local area in natural images. Compared with the conventional prediction methods with fixed predictors based on the arithmetic combination of neighboring pixels, the proposed prediction method acquires better accuracy and more concentrated prediction-error histogram for reversible data embedding. Experimental results demonstrate that the proposed method achieves superior performance compared with some previously reported methods.

Another paper entitled "Overlapping Pixel Value Ordering Predictor for High-Capacity Reversible Data Hiding", co-authored by Chin-Feng Lee, Jau-Ji Shen, Yu-Chi Kao, and Somya Agrawal, focuses on the overlapping pixel value ordering (OPVO) method for reversible data hiding to obtain high-quality camouflage images under good embedding rate. In this method, secret bits are embedded in the maximum and minimum pixel values, and each pixel can embed data multiple times. The correlation between adjacent pixels in the natural image is exploited to improve hiding capacity. As a result, the hiding capacity of the proposed OPVO method is more than twice as high as that of the previous PVO-based methods under the condition of acceptable stego-image quality.

In the paper entitled "A Code Division Multiplexing and Block Classification-Based Real-Time Reversible 
Data-Hiding Algorithm for Medical Images”, Bin Ma, Bing Li, Xiaoyu Wang, Chunpeng Wang, Jian Li, and Yunqing Shi present a RDH scheme for medical images in the wavelet domain. In this scheme, image blocks are classified into smooth and textural groups, and the wavelet sub-bands of textural blocks are utilized for secret data embedding through the strategy of code division multiplexing (CDM). Moreover, because the procedure of secret data embedding is associated with some parameters, thus, the receiver without knowing correct parameters cannot reconstruct the secret data and the cover image reversibly. Experimental results show that the proposed RDH scheme achieves better ratedistortion performance than some state-of-the-art schemes, especially for medical images, and can guarantee a satisfactory data transmission security.

\section{Emerging technique}

Visual secret sharing is an effective technique for the protection of data transmission. In order to enhance the security, the data hiding-based visual secret sharing (DH-VSS) methods are emerging and have attracted considerable research attentions recently. In the paper entitled "Real-Time Adaptive Visual Secret Sharing with Reversibility and High Capacity", Ching-Chun Chang, Yanjun Liu, and Kaimeng Chen propose a high-capacity $(2,2)$ DH-VSS method based on the LSB substitution technique, which includes two stages, i.e., shadow creation and secret extraction and image reconstruction. In the first stage, original cover image is divided into eight bit planes and secret data are embedded in the 4-LSB bit planes to generate two shadow images. In the second stage, both the embedded data and the cover image can be correctly recovered with two shadows. Experimental results demonstrate that this method has satisfactory performance of computational complexity and embedding capacity.

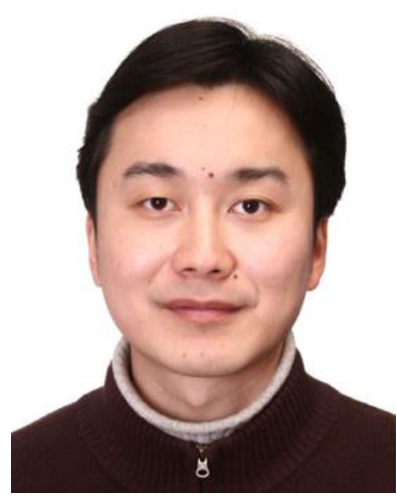

Chuan Qin received the B.S. degree in electronic engineering and the M.S. degree in signal and information processing from Hefei University of Technology, Anhui, China, in 2002 and 2005, respectively, and the Ph.D. degree in signal and information processing from Shanghai University, Shanghai, China, in 2008. Since 2008, he has been with the faculty of the School of Optical-Electrical and Computer Engineering, University of Shanghai for Science and Technology, where he is currently a Professor. He was with Feng Chia University at Taiwan as a Postdoctoral Researcher from July 2010 to July 2012. His research interests include image processing and multimedia security. He has published over 120 papers in these research areas.

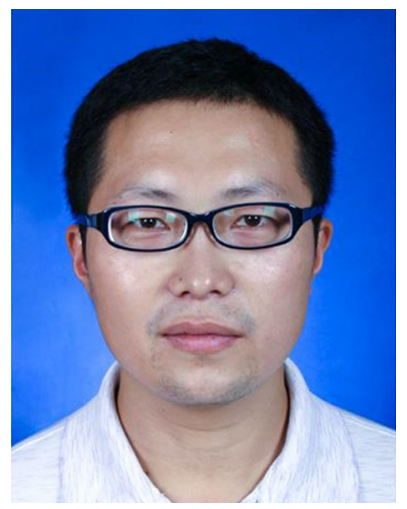

Zhenxing Qian received both the B.S. and the Ph.D. degrees from University of Science and Technology of China (USTC) in 2003 and 2007, respectively. Since 2009 , he has been with the faculty of the School of Communication and Information Engineering, Shanghai University. Now, he is a Professor of Fudan University. He has published over 80 peer-reviewed papers on international journals and conferences, and has managed more than 10 projects including three projects of National Science

Foundation of China and the Shanghai Rising-Star Project His research interests include information hiding, image processing and multimedia security.

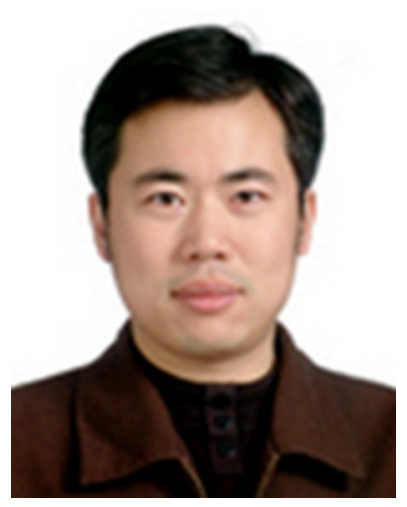

Guorui Feng received the B.S. and M.S. degree in computational mathematic from Jilin University, China, in 1998 and 2001 respectively. He received $\mathrm{Ph} . \mathrm{D}$. degree in electronic engineering from Shanghai Jiaotong University, China, 2005. From January 2006 to December 2006, he was an assistant professor in East China Normal University, China. During 2007, he was a research fellow in Nanyang Technological University, Singapore. Now he is with the School of Communication and Information Engineering, Shanghai University, China, where he is currently a Professor. His current research interests include image processing, image analysis and computational intelligence.

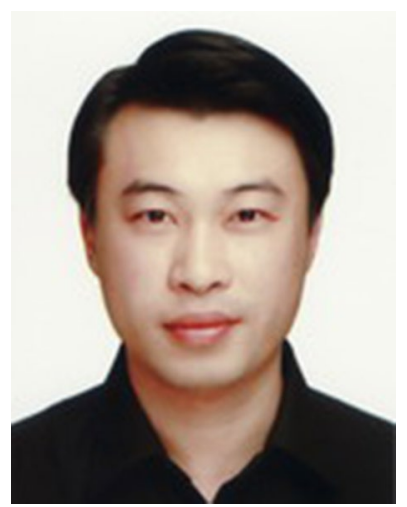

Xinpeng Zhang received the B.S. degree in computational mathematics from Jilin University, China, in 1995, and the M.E. and Ph.D. degrees in communication and information system from Shanghai University, China, in 2001 and 2004, respectively. Since 2004, he has been with the faculty of the School of Communication and Information Engineering, Shanghai University. Now, he is a Professor of Fudan University. He was with the State University of New York at Binghamton as a visiting scholar from January 2010 to January 2011, and Konstanz University as an experienced researcher sponsored by the Alexander von Humboldt Foundation from March 2011 to May 2012. He is an Associate Editor for the IEEE Transactions on Information Forensics and Security. His research interests include multimedia security, image processing, and digital forensics. He has published more than 200 papers in these areas. 